\title{
Expectation and Emotion Enhancement Using Content Knowledge in Cultural Experiences: An Abstract
}

\author{
Nadia Steils, Zakia Obaidalahe, Dominique Crié, and Francis Salerno
}

\begin{abstract}
This research aims to understand how providing consumers with knowledge about the content of a cultural experience in advance shapes their expectations and primes anticipated emotions, which in turn affects consumers' overall experience. We used theater plays as a context for this study. Using an exploratory qualitative and two quantitative field studies, our findings support that delivering content knowledge about a performance in advance has a direct influence on visitors' cultural experience before (rational expectations, anticipated emotions, intention to attend the performance) and an indirect influence after the experience (affective reactions and satisfaction). Our results show that rational and affective expectations are forged differently. Due to the uniqueness of each performance, rational expectations are created based on the information gathered by individuals in advance, as no comparison standards exist, while affective expectations (i.e., anticipated emotions) are primed and risk reducing visitors' satisfaction if these affective expectations are set too high. The study's results provide support for the use of detailed information brochures to shape, guide, and enhance consumers' cultural experiences. In contrast with early research suggesting separating marketing approaches from cultural experiences, we show that consumers' expectations and thus a customer-centric approach should not further be neglected.
\end{abstract}

\footnotetext{
N. Steils $(\bowtie)$

University of Namur, Namur, Belgium

IAE Lille, Lille, France

e-mail: nadia.steils@unamur.be

Z. Obaidalahe $\bullet$ D. Crié $\bullet$ F. Salerno

IAE Lille, Lille, France

e-mail: obaidalahe.zakia@gmail.com; dominique.crie@univ-lille1.fr; francis.salerno@

univ-lille1.fr
} 